\title{
Whole-transcriptome sequencing identified gene expression signatures associated with aggressive clear cell renal cell carcinoma
}

\author{
Ken Batai ${ }^{1}$, Elliot Imler ${ }^{2}$, Jayce Pangilinan ${ }^{1}$, Robert Bell ${ }^{3}$, Aye Lwin ${ }^{1}$, Elinora Price ${ }^{4}$, \\ Tijana Milinic ${ }^{1}$, Amit Arora ${ }^{5}$, Nathan A. Ellis ${ }^{6}$, Erika Bracamonte ${ }^{3}$, Bruce Seligmann ${ }^{2}$ \\ and Benjamin R. Lee ${ }^{1}$ \\ ${ }^{1}$ Division of Urology, Department of Surgery, University of Arizona, Tucson, AZ, USA \\ 2 BioSpyder Technologies, Inc, Tucson, AZ, USA \\ ${ }^{3}$ Department of Pathology, University of Arizona, Tucson, AZ, USA \\ ${ }^{4}$ Department of Surgery, University of Arizona, Tucson, AZ, USA \\ ${ }^{5}$ Department of Epidemiology and Biostatistics, University of Arizona, Tucson, AZ, USA \\ ${ }^{6}$ Department of Cellular and Molecular Medicine, University of Arizona, Tucson, AZ, USA \\ Correspondence to: Ken Batai, email: kbatai@arizona.edu \\ Keywords: kidney cancer; biomarkers; molecular subtype; Hispanic Americans; whole transcriptome sequencing \\ Received: September 24, $2018 \quad$ Accepted: October 31,2018 Published: Novembr 11, 2018
}

Copyright: Batai et al. This is an open-access article distributed under the terms of the Creative Commons Attribution License 3.0 (CC BY 3.0), which permits unrestricted use, distribution, and reproduction in any medium, provided the original author and source are credited.

\section{ABSTRACT}

Clear cell renal cell carcinoma (cCRCC) is the most prevalent subtype of kidney cancer, yet molecular biomarkers have not been used for the prognosis of ccRCC to aide clinical decision making. This study aimed to identify genes associated with cCRCC aggressiveness and overall survival (OS). Samples of ccRCC tumor tissue were obtained from 33 patients who underwent nephrectomy. Gene expression was determined using whole-transcriptome sequencing. The Cancer Genome Atlas Kidney Renal Clear Cell Carcinoma (TCGA-KIRC) RNA-seq data was used to test association with OS. 290 genes were differentially expressed between tumors with high and low stage, size, grade, and necrosis (SSIGN) score ( $\geq 7$ vs. $\leq 3$ ) with $P_{A D J}<0.05$. Four genes, G6PD, APLP1, GCNT3, and PLPP2, were also over-expressed in advanced stage (III and IV) and high grade ( 3 and 4$)$ CCRCC and tumor with necrosis $\left(P_{A D J}<0.05\right)$. Investigation stratifying by stage found that APLP1 and PLPP2 overexpression were significantly associated with poorer OS in the early stage (Quartile 1 vs. Quartile 4, HR = 3.87, 95\% CI:1.25-11.97, P = 0.02 and HR = 4.77, 95\% CI:1.37-16.57, P = 0.04 respectively). These genes are potential biomarkers of ccRCC aggressiveness and prognosis that direct clinical and surgical management.

\section{INTRODUCTION}

Kidney cancer is among the 10 most common cancers [1], and both globally and nationally, its incidence has significantly increased over the past 30 years since the 1980s [2]. Currently, prognosis of renal cell carcinoma (RCC) and post-surgical surveillance strategies are mainly based on staging and histology. A composite score combining multiple factors including stage, size, grade, and necrosis (SSIGN) score may be useful for predicting prognosis [3, 4], but molecular biomarkers prognostic of local recurrence also helps develop individuallytailored clinical management strategies before or after nephrectomy.

Several gene expression studies have identified molecular subtypes within RCC, and within the most common subtype of RCC, clear cell renal carcinoma (ccRCC) [5, 6]. Patients with ccRCC molecular subtype $\mathrm{ccB}$ (CC-e.3) have worse survival rates than patients with subtype ccA (CC-e.1 and CC-e.2) [5, 6]. Another study demonstrated that molecular subtypes are correlated with SSIGN score [7]. Gene expression studies also 
identified some genes that predict ccRCC aggressiveness and progression [8-11]. However, currently there is no verified set of molecular biomarkers that can be used in a clinical setting to move toward precision medicine of RCC treatment, much less such a signature measured from formalin-fixed paraffin embedded (FFPE) on a platform that can be used clinically. Moreover, Hispanic Americans (HAs) and Native American have been found to have a higher burden of kidney cancer, but they are often underrepresented in the molecular studies [12, 13]. Including the high risk previously underrepresented populations may help identify novel molecular signatures.

In this study, we measured gene expression profiles of ccRCC directly from FFPE cores to identify molecular subtypes and gene expression signatures that were associated with ccRCC aggressiveness as assessed by tumor characteristics, including the SSIGN score. A novel extraction-free whole transcriptome sequencing method (TempO-Seq ${ }^{\mathrm{TM}}$ ) was used to measure gene expression. Then, The Cancer Genome Atlas Kidney Renal Clear Cell Carcinoma (TCGA-KIRC) RNA-seq data was used to verify the findings and test association between identified gene expression signatures and overall survival (OS).

\section{RESULTS}

\section{Patients' characteristics}

Table 1 shows that characteristics of 33 patients included in this study. The mean age of patients was 56.7, and about half of patients had advanced stage ccRCC $(54.5 \%)$ with 7 patients $(21.2 \%)$ who had metastasis at presentation. Necrosis was found in tumor from 15 patients (48\%). While 14 patients $(42.4 \%)$ had low SSIGN score, 12 patients $(36.4 \%)$ had high SSIGN score. We observed 6 recurrences and 4 deaths during the follow-up period (median follow-up days of 908 for recurrence and 932 for mortality). Of 4 patients who died, 3 died from disease specific cause and one died from complication related to surgery. Of 33 patients, 15 were EAs and 15 were HAs. HA patients and patients from unknown racial/ ethnic background were younger than EA patients (mean age of 54.3 in HAs compared to 60.4 in EAs). Compared to HAs, EA patients had significantly higher creatinine levels $(P=0.01)$. Metastasis and high-grade tumor was slightly more common in EAs than HAs $(33.3 \%$ vs $13.3 \%$ for metastasis at presentation and $73.3 \%$ vs. $66.7 \%$ for Fuhrman Grade). EAs and other racial/ethnic groups were similar in other aspects.

\section{Molecular subtype}

The TempO-Seq data successfully grouped 32 out of 33 patients into previously defined molecular subtypes
(ccA and ccB) (Supplementary Figure 1). ccA and ccB subtype could not be assigned to one HA patient with advanced stage ccRCC. ccB patients were significantly older $(P=0.04)$ and had higher creatinine levels $(P=0.03)$ than patients with ccA subtype (Table 2$)$. The molecular subtype ccB was found in $58.3 \%$ of patients with higher SSIGN score $(\geq 7)$, while $42.9 \%$ of patients with low SSIGN score $(\leq 3)$ had $c c B$. The subtype $c c B$ was more common in patients with Fuhrman Grade 3 or $4(50.0 \%)$, metastasis $(57.1 \%)$, and tumor necrosis $(60.0 \%)$ than patients with Furman Grade 1 or $2(30.0 \%)$ and patients without metastasis $(40.0 \%)$ and tumor necrosis $(31.3 \%)$. Patients with subtype ccB also had larger tumor size than patients with subtype ccA (mean, 7.5 vs. $5.8 \mathrm{~cm}$ ). ccB was more common among patients who had recurrence $(66.7 \%)$ than who did not (38.5\%). Moreover, all the patients who passed away had ccB subtype. Molecular subtype, ccA, was more common in HAs than EAs $(64.3 \%$ vs. $41.2 \%)$.

\section{Differentially expressed genes between aggressive and non-aggressive ccRCC}

We performed two step analysies to identify differentially expressed genes between aggressive and nonaggressive ccRCC. First, comparing patients with low vs high SSIGN score ( $\leq 3 v s . \geq 7)$ identified 590 differentially expressed genes $\left(P_{\mathrm{ADJ}}<0.05\right)$ (Supplementary Figure 2). 281 genes were over-expressed and 309 genes were downregulated in the ccRCC with high SSIGN score. Then, we performed analysis to identify genes that were also differentially expressed 1) between advanced and early stage, 2) between high and low grade, and 3) between tumors with necrosis and without. Of these 590 genes, only 4 genes (APLP1, G6PD, GCNT3, and PLPP2) were consistently differentially expressed, and they were overexpressed in advanced stage and high-grade tumors as well as tumors with necrosis $\left(P_{\mathrm{ADJ}}<0.05\right)$ (Table 3$)$. The most strongly over-expressed gene was amyloid beta precursor like protein 1, APLP1. APLP1 had 2.8-fold higher expression in tumor with high compared to low SSIGN score $\left(P_{\mathrm{ADJ}}=4.4 \times 10^{-6}\right)$. APLP1 also had 2.4-fold higher expression in advanced stage ccRCC and 2.6-fold higher expression in high-grade ccRCC. However, although trending for significance, expression difference between tumor and normal samples for $A P L P 1$ was not statistically significant after adjusting for multiple comparison $\left(P_{\text {ADJ }}\right.$ $=0.06$ ), while three other genes were over-expressed in tumor compared to normal tissue samples $\left(P_{\mathrm{ADJ}}<0.05\right)$.

Among genes implicated in $\mathrm{RCC}, B A P 1$ and SETD2 were down regulated in aggressive ccRCC $(P<$ 0.05), but other genes, PBRM1, KDM5C, VHL, PTEN, and $M T O R$, were not associated with aggressive ccRCC (Supplementary Table 1). We also evaluated differential expression between high and low SSIGN score for 64 


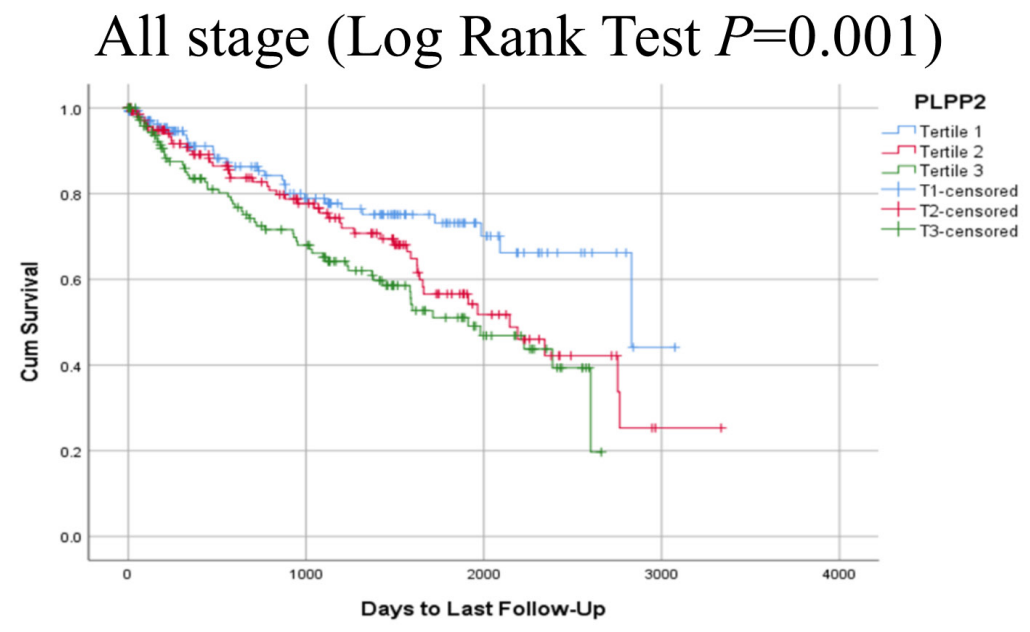

Early Stage (Log Rank Test $P=0.047$ )

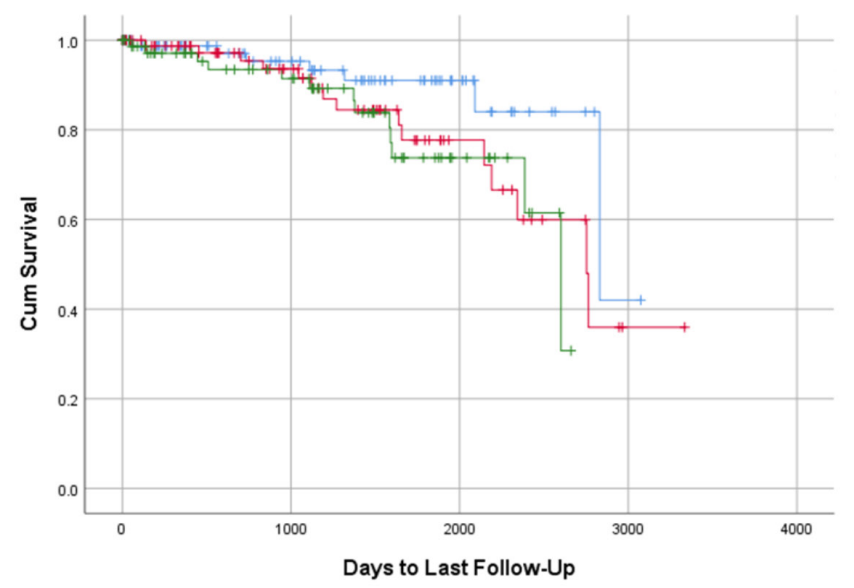

\section{Advanced Stage (Log Rank Test $P=0.16)$}

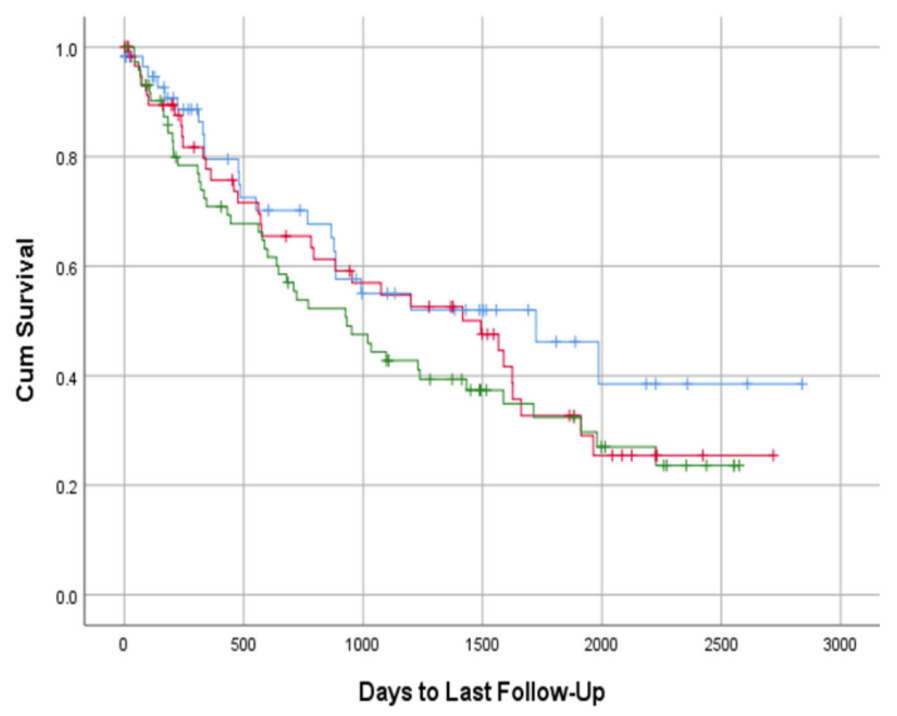

Figure 1: Kaplan-Meier plots for the PLPP2 gene. A. all stages (Log Rank Test $P=0.001)$, B. early stage (Log Rank Test $P=$ 0.047), and C. advanced stage (Log Rank Test $P=0.16$ ). 
Table 1: Patients characteristics

\begin{tabular}{|c|c|c|c|c|}
\hline & Total $(n=33)$ & $\operatorname{EAs}(n=15)$ & HAs $(n=15)$ & unknown $(n=3)$ \\
\hline Age, mean (SD) & $56.7(13.6)$ & $60.4(15.1)$ & $54.3(11.7)$ & $49.7(13.2)$ \\
\hline \multicolumn{5}{|l|}{ Gender, $n(\%)$} \\
\hline Female & $15(45.5 \%)$ & $7(46.7 \%)$ & $6(40.0 \%)$ & $2(66.7 \%)$ \\
\hline Male & $18(54.5 \%)$ & $8(53.3 \%)$ & $9(60.0 \%)$ & $1(33.3)$ \\
\hline \multicolumn{5}{|l|}{ Body Mass Index, $n(\%)$} \\
\hline Normal $(<30)$ & $19(57.6)$ & $9(60.0)$ & $9(60.0)$ & $1(33.3)$ \\
\hline Obese $(\geq 30)$ & $14(42.4)$ & $6(40.0)$ & $6(40.0)$ & $2(66.7)$ \\
\hline \multicolumn{5}{|l|}{ Diabetes, $n(\%)$} \\
\hline No & $22(66.7)$ & $10(66.7)$ & $11(73.3)$ & $1(33.3)$ \\
\hline Yes & $11(33.3)$ & $5(33.3)$ & $4(26.7)$ & $2(66.7)$ \\
\hline \multicolumn{5}{|l|}{ Hypertension, $n(\%)$} \\
\hline No & $13(39.4 \%)$ & $5(33.3 \%)$ & $6(40.0 \%)$ & $2(66.7 \%)$ \\
\hline Yes & $20(60.6 \%)$ & $10(66.7 \%)$ & $9(60.0 \%)$ & $1(33.3 \%)$ \\
\hline Creatinine (mg/dL), Median (IQR) & $0.80(0.80-1.00)$ & $0.90(0.85-1.20)$ & $0.80(0.70-0.90)^{*}$ & $0.80(0.80-0.80)$ \\
\hline \multicolumn{5}{|l|}{ GFR $(\mathrm{ml} / \mathrm{min} / 1.73 \mathrm{~cm} 2), n(\%)$} \\
\hline$<60$ & $4(12.9 \%)$ & $2(15.4 \%)$ & $2(13.3 \%)$ & $0(0.0 \%)$ \\
\hline$>60$ & $27(87.1 \%)$ & $11(84.6 \%)$ & $13(86.7 \%)$ & $3(100.0 \%)$ \\
\hline \multicolumn{5}{|l|}{ TNM Stage, $n(\%)$} \\
\hline I or II & $15(45.8 \%)$ & $7(46.7 \%)$ & $7(46.7 \%)$ & $1(33.3)$ \\
\hline III or IV & $18(54.5 \%)$ & $8(53.3 \%)$ & $8(53.3 \%)$ & $2(66.7)$ \\
\hline \multicolumn{5}{|l|}{ Tumor Stage, n (\%) } \\
\hline T1 & $14(42.4 \%)$ & $7(46.7 \%)$ & $6(40.0 \%)$ & $1(33.3 \%)$ \\
\hline T2 & $1(3.0 \%)$ & $0(0.0 \%)$ & $1(6.7 \%)$ & $0(0.0 \%)$ \\
\hline T3 & $17(51.5 \%)$ & $7(46.7 \%)$ & $8(53.3 \%)$ & $2(66.7 \%)$ \\
\hline T4 & $1(3.0 \%)$ & $1(6.7 \%)$ & $0(0.0 \%)$ & $0(0.0 \%)$ \\
\hline \multicolumn{5}{|l|}{ Regional Lymph Nodes, $n(\%)$} \\
\hline No & $30(90.9 \%)$ & $13(86.7 \%)$ & $14(93.3 \%)$ & $3(100.0 \%)$ \\
\hline Yes & $3(9.1 \%)$ & $2(13.3 \%)$ & $1(6.7 \%)$ & $0(0.0 \%)$ \\
\hline \multicolumn{5}{|l|}{ Metastasis, $n(\%)$} \\
\hline No & $26(78.8 \%)$ & $10(66.7 \%)$ & $13(86.7 \%)$ & $3(100.0 \%)$ \\
\hline Yes & $7(21.2 \%)$ & $5(33.3 \%)$ & $2(13.3 \%)$ & $0(0.0 \%)$ \\
\hline \multicolumn{5}{|l|}{ Fuhrman Grade, $n(\%)$} \\
\hline 1 or 2 & $10(30.3 \%)$ & $4(26.7 \%)$ & $5(33.3 \%)$ & $1(33.3 \%)$ \\
\hline 3 or 4 & $23(69.7 \%)$ & $11(73.3 \%)$ & $10(66.7 \%)$ & $2(66.7 \%)$ \\
\hline \multicolumn{5}{|l|}{ Necrosis, $n(\%)$} \\
\hline No & $16(51.6 \%)$ & $7(50 \%)$ & $7(50 \%)$ & $2(66.7 \%)$ \\
\hline Yes & $15(48.4 \%)$ & $7(50 \%)$ & $7(50 \%)$ & $1(33.3 \%)$ \\
\hline Tumor Size (diameter, cm), mean (SD) & $6.5(3.9)$ & $5.9(3.4)$ & $7.3(4.6)$ & $5.3(1.1)$ \\
\hline \multicolumn{5}{|l|}{ SSIGN, $n(\%)$} \\
\hline Low $(\leq 3)$ & $14(42.4 \%)$ & $7(46.7 \%)$ & $5(33.3 \%)$ & $2(66.7 \%)$ \\
\hline Intermediate (4-6) & $7(21.2 \%)$ & $2(13.3 \%)$ & $4(26.7 \%)$ & $1(33.3 \%)$ \\
\hline High $(\geq 7)$ & $12(36.4 \%)$ & $6(40.0 \%)$ & $6(40.0 \%)$ & $0(0.0 \%)$ \\
\hline \multicolumn{5}{|l|}{ Negative/Positive Margins, $n(\%)$} \\
\hline Positive & $9(27.3 \%)$ & $5(33.3 \%)$ & $4(26.7 \%)$ & $0(0.0 \%)$ \\
\hline Negative & $24(72.7 \%)$ & $10(66.7 \%)$ & $11(73.3 \%)$ & $3(100.0 \%)$ \\
\hline \multicolumn{5}{|l|}{ Recurrence, $n(\%)$} \\
\hline No & $27(81.8 \%)$ & $12(80.0 \%)$ & $12(80.08 \%)$ & $3(100.0 \%)$ \\
\hline Yes & $6(18.2 \%)$ & $3(20.0 \%)$ & $3(20.0 \%)$ & $0(0.0 \%)$ \\
\hline \multicolumn{5}{|l|}{ Vital Status, $n(\%)$} \\
\hline Alive & $29(87.9 \%)$ & $14(93.3 \%)$ & $12(80.0 \%)$ & $3(100.0 \%)$ \\
\hline Deceased & $4(12.1 \%)$ & $1(6.7 \%)$ & $3(20.0 \%)$ & $0(0.0 \%)$ \\
\hline
\end{tabular}

* $P=0.01$ (Independent Sample Mann-Whitney U-Test) 
Table 2: Molecular subtypes (ccA vs. ccB)

\begin{tabular}{|c|c|c|}
\hline & $\operatorname{ccA}(n=18)$ & $\operatorname{ccB}(n=14)$ \\
\hline Age, mean (SD) ${ }^{1}$ & $52.2(15.8)$ & $61.4(7.8)$ \\
\hline \multicolumn{3}{|l|}{ Gender, $n(\%)$} \\
\hline Male & $7(41.2 \%)$ & $10(58.8 \%)$ \\
\hline Female & $11(73.3 \%)$ & $4(26.7 \%)$ \\
\hline \multicolumn{3}{|l|}{ Race/ethnicity, $n(\%)$} \\
\hline European Americans & $6(40.0 \%)$ & $9(60.0 \%)$ \\
\hline Hispanic Americans & $9(64.3 \%)$ & $5(35.7 \%)$ \\
\hline Others & $3(100.0 \%)$ & \\
\hline Creatinine, Median (IQR) ${ }^{2}$ & $0.80(0.70-0.90)$ & $0.95(0.80-1.20)$ \\
\hline \multicolumn{3}{|l|}{ GFR, $n(\%)$} \\
\hline$<60$ & $2(50.0 \%)$ & $2(50.0 \%)$ \\
\hline$>60$ & $16(61.5 \%)$ & $10(38.5 \%)$ \\
\hline \multicolumn{3}{|l|}{ Fuhrman Grade, $n(\%)$} \\
\hline 1 or 2 & $7(70.0 \%)$ & $3(30.0 \%)$ \\
\hline 3 or 4 & $11(50.0 \%)$ & $11(50.0 \%)$ \\
\hline \multicolumn{3}{|l|}{ TNM Stage, $n(\%)$} \\
\hline I or II & $9(60.0 \%)$ & $6(40.0 \%)$ \\
\hline III or IV & $9(52.9 \%)$ & $8(47.1 \%)$ \\
\hline \multicolumn{3}{|c|}{ Metastasis at Presentation, $n(\%)$} \\
\hline No & $15(60.0 \%)$ & $10(40.0 \%)$ \\
\hline Yes & $3(42.9 \%)$ & $4(57.1 \%)$ \\
\hline \multicolumn{3}{|l|}{ Necrosis, $n(\%)$} \\
\hline No & $11(68.8 \%)$ & $5(31.3 \%)$ \\
\hline Yes & $6(40.0 \%)$ & $9(60.0 \%)$ \\
\hline Tumor Size, mean (SD) & $5.8(2.9)$ & $7.5(4.8)$ \\
\hline \multicolumn{3}{|l|}{ SSIGN, $n(\%)$} \\
\hline Low $(\leq 3)$ & $8(57.1 \%)$ & $6(42.9 \%)$ \\
\hline $\operatorname{High}(\geq 7)$ & $5(41.7 \%)$ & $7(58.3 \%)$ \\
\hline \multicolumn{3}{|l|}{ Recurrence, $n(\%)$} \\
\hline No & $16(61.5 \%)$ & $10(38.5 \%)$ \\
\hline Yes & $2(33.3 \%)$ & $4(66.7 \%)$ \\
\hline \multicolumn{3}{|l|}{ Vital Status, $n(\%)^{3}$} \\
\hline Alive & $18(64.3 \%)$ & $10(35.7 \%)$ \\
\hline Deceased & $0(0.0 \%)$ & $4(100.0 \%)$ \\
\hline
\end{tabular}

${ }^{1} P=0.04$ (Independent Sample T-test)

${ }^{2} P=0.03$ (Independent Sample Mann-Whitney U-Test)

${ }^{3} P=0.04$ (Chi-Square Test)

genes associated with ccRCC aggressiveness, recurrence, or survival identified in previous studies [8, 9, 11, 18-21]. 34 genes were validated with nominal $P<0.05$, and 19 genes, including S1PR1, TSPAN7, EDNRB, had $P_{\text {ADJ }}<$ 0.05 (Supplementary Table 2).

We investigated if the expression of $A P L P 1$, $G 6 P D, G C N T 3$, and PLPP2 were correlated with other clinical characteristics and outcomes (Supplementary Table 3). Correlation between G6PD expression and preoperative creatinine levels was statistically significant (Spearman's rho 0.423, $P=0.02$ ). APLP1 and G6PD were overexpressed in patients without metastatic ccRCC at presentation who had recurrence later $(P=0.03$ and
$P=0.01$ respectively). We observed significantly higher expression of APLP1, G6PD, and PLPP2 in patients who died during the follow-up $(P<0.05)$. GCNT3 was overexpressed in tumors from deceased patients, but it was not statistically significant $(P=0.06)$

We further explored the gene expression of the 4 identified genes in the TCGA-KIRC dataset to validate our findings (Supplementary Table 4). All 4 genes were overexpressed in advanced stage compared to early stage ccRCC $(P<0.05)$, and G6PD, APLP1 and GCNT3 were overexpressed in high grade compared to low grade ccRCC $(P<0.05)$. G6PD, APLP1, and PLPP2 were overexpressed in ccRCC with necrosis $(P<0.05)$. 
Table 3: Genes overexpressed ( $\log _{2}$ fold change) in aggressive ccRCC

\begin{tabular}{|l|l|c|c|c|c|}
\hline \multicolumn{1}{|c|}{ Gene } & \multicolumn{1}{|c|}{ Gene Name } & FC (SSIGN) & FC (Stage) & FC (Grade) & FC (Necrosis) \\
\hline APLP1 & amyloid beta precursor like protein 1 & 2.8 & 2.4 & 2.6 & 1.9 \\
\hline G6PD & glucose-6-phosphate dehydrogenase & 2.5 & 2.0 & 1.6 & 2.8 \\
\hline GCNT3 & glucosaminyl (N-acetyl) transferase 3, mucin type & 2.3 & 2.1 & 2.1 & 2.3 \\
\hline $\boldsymbol{P L P P 2}$ & phospholipid phosphatase 2 & 2.3 & 1.9 & 1.9 & 2.4 \\
\hline
\end{tabular}

However, only $G 6 P D$ was significantly over-expressed in tumor compared to normal tissues $(P=0.001) . P L P P 2$ gene was over-expressed in tumor, but was not statistically significant $(P=0.07)$. On the other hand, APLP1 was over-expressed in normal compared to tumor samples $(P<0.001)$. When expression levels in normal samples were examined, significantly higher expression of $G 6 P D$ and $P L P P 2$ was observed in patients with advanced stage ccRCC compared to early stage ccRCC $(P<0.05)$. No significant difference in gene expression was observed for grade and necrosis in normal tissues.

\section{Survival analysis with TCGA-KIRC dataset}

In an unadjusted model, expression of $A P L P 1$, $G 6 P D$, and $P L P P 2$ were significantly associated with OS in the TCGA-KIRC dataset (Supplementary Figure 3, Supplementary Table 5). When we stratified the patients by stage, $P L P P 2$ expression showed significant association with OS in the early stage group (Log Rank Test $P=$ 0.047), but not in the advanced stage group (Figure 1). We did not see this pattern of differential expression for any of the other genes.

In Cox regression analysis, higher expression of $A P L P 1, G 6 P D$ and $P L P P 2$ were associated with poorer OS in an unadjusted model (Quartile $1 v s$. Quartile 4, HR $=2.78,95 \%$ CI:1.65-4.68, HR $=1.99,95 \%$ CI:1.26-3.15, and HR $=2.27,95 \%$ CI:1.38-3.72, respectively, all $P<$ $0.001)$. Once the model was adjusted, these associations were no longer significant. Further investigation stratifying by stage found that $A P L P 1$ and $P L P P 2$ overexpression were significantly associated with poorer OS in the early stage (Quartile 1 vs. Quartile 4, HR $=3.87,95 \%$ CI:1.2511.97, $P=0.02$ and HR $=4.77,95 \%$ CI:1.37-16.57, $P$ $=0.04$ respectively) in an adjusted model. Linear trend of association was significant for $A P L P 1$ expression $(P=$ $0.006)$ and approaching significance for $P L P P 2$ expression $(P=0.06)$.

\section{DISCUSSION}

Previous gene expression studies identified some biomarkers and signatures that are associated with aggressive ccRCC or ccRCC prognosis and survival [5-
11]. However, these studies mainly included European populations. With over-representation of HAs, this study attempted to identify novel molecular signatures of aggressive ccRCC. It was in the analysis of the association of differential gene expression with SSIGN score that we made our most significant observations and found 4 genes that were over-expressed in tumors with high SSIGN score as well as advanced stage and high grade ccRCC and ccRCC with necrosis. Of 4 genes, 2 of them, APLP1 and $P L P P 2$, were also associated with OS in early stage ccRCC.

$A P L P 1$, involved in glucose homeostasis, was a novel finding showing strong association with OS in ccRCC but was over-expressed in normal compared to tumor samples $(P<0.001)$. APLP1 is closely related to $A P P$ and $A P L P 2$, both of which are associated with aggressive cancers in other organs [22]. PLPP2, was overexpressed in advanced stage and high-grade tumors and tumors with necrosis, and was also associated with OS in patients with early stage ccRCC. PLPP2 is a gene in the phosphatidic acid phosphatase (PAP) family and a key enzyme in lipid metabolism. Lipid Phosphate Phosphatases are likely to be involved in cancer cell proliferation [23]. Other genes that topped the list of differential expression were $G 6 P D$ and GCNT3. Association between expression of G6PD, glucose-6-phosphate dehydrogenase, and aggressiveness was validated using the TCGA KIRC dataset. $G 6 P D$ is an enzyme involved in carbohydrate metabolism, conversion of glucose to ribose-5-phosphate. The previous studies show that $G 6 P D$ is involved in RCC proliferation and associated with survival [24, 25], and is a drug resistance gene in colon cancer [26]. Another gene, GCNT3, glucosaminyl (N-acetyl) transferase 3, is involved in colon and pancreatic cancer [27, 28].

Of these genes, only G6PD were validated with the TCGA dataset using our criteria. It is possible that it was due to small sample size in our study, but it should also be noted that discovery samples we used had different demographic characteristics than the TCGA data. In the TCGA data, a large majority of samples $(82.7 \%)$ were individuals of European descent, while our discovery data composed of only $45.5 \%$ EAs. It is likely that HAs and other racial/ethnic groups have very different behavioral and social factors that contributed to tumor characteristics at diagnosis. For example, genes associated 
with metabolism, G6PC, PLPP2, and APLP1, were overexpressed in advanced stage and high grade ccRCC. Obesity is associated with higher kidney cancer mortality [29], and distribution of adiposity or body composition varies across racial/ethnic groups [30]. Obesity may affect ccRCC progression through different mechanisms in EAs and HAs. Obesity and other behavioral factors may also affect tumor microenvironment [31, 32], and G6PD and PLPP2 were also overexpressed in normal tissues form patients with advanced stage ccRCC in the TCGA dataset. In a follow-up study, we will assess the relationships between obesity and molecular biomarkers in ccRCC progression. Besides these four genes, additional sets of genes associated with ccRCC progression in other studies were also associated with SSIGN score, providing a molecular signature for this clinical assessment score.

There are several major limitations of current study. First, small sample size was very small in the initial gene expression analysis, so we were not able to perform analysis adjusting for relevant variables. Also, we may have observed false positive signatures, which could not be verified using the TCGA data. Moreover, sample size of our discovery dataset did not allow us to fully investigate the causal relationship between gene expression signatures and ccRCC aggressiveness. To test the causal relationship, we used the TCGA KIRC dataset to test if expression of these genes is associated with OS. Of 4 genes identified, only G6PD was validated and APLP1 and PLPP2 were associated with OS. The difference between our discovery and the TCGA data set could result from the difference in sensitivity and specificity of the TempO-Seq platform compared to RNA-sequencing in the TCGA data, difference patient characteristics, and sample size. Lastly, it could also have been due to the physical differences in the samples used. We obtained $1 \mathrm{~mm}$ punches of FFPE with about $5 \mathrm{~mm}$ thickness, and assayed the entire sample. The TGCA data set used RNA/DNA co-extracted from $30 \mathrm{mg}$ of frozen surgical resections. Tumor tissue from a single patient can be very heterogeneous, such that using a large amount of sample may have impacted study findings. None-the-less, to develop a clinically useful test, it is necessary to test FFPE, which is one reason why we set out to demonstrate the ability to do so in this study. We demonstrated that the TempO-Seq assay from FFPE samples can identify previously reported gene expression signatures and novel signatures associated with high SSIGN score and OS. In the follow-up study, we will test with a larger sample to permit finer-scale molecular subtype analysis and race/ethnicity stratified analysis; 1) using $1 \mathrm{~mm}^{2}$ areas of $>90 \%$ cancer tissue for assay; and 2) taking advantage of an advance in the TempO-Seq technology (Digital Spatial Molecular Profiling) which permits even smaller areas of tissue to be profiled and correlated directly to the morphology of the tissue.

\section{MATERIALS AND METHODS}

\section{Samples}

After Institutional Review Board approval, the clinical information of RCC patients, who underwent robotic, laparoscopic or open partial or radical nephrectomy at our University hospital between 2010 and 2016 were reviewed $(n=361), 51$ samples were selected and reviewed by board certified genitourinary pathologists. The final set of 33 patients who were diagnosed with ccRCC and had no previous diagnosis of cancer were selected based on staging, with close to equal representation of HA and European American (EA) patients. Patients' demographic (age at surgery, gender, and race/ethnicity) and clinical information, including pre-operation creatinine levels, glomerular filtration rate (GFR), histological subtype, stage at diagnosis, Fuhrman Grade, tumor size, and presence of necrosis, was obtained from their electronic medical record. The Stage, Size, Grade, and Necrosis (SSIGN) score [3, 4], a composite score of these 4 clinical assessment measures, was used to define aggressiveness of ccRCC; non-aggressive $(\mathrm{SSIGN} \leq 3)$, intermediate (SSIGN between 4 and 6$)$, and aggressive (SSIGN $\geq 7$ ).

Corresponding Hematoxylin and Eosin (H\&E) stained slides were retrieved for each patient identified, and each slide was reviewed by a board-certified pathologist and pathology resident. Slides were evaluated to identify areas of highest tumor grade of RCC, with morphologic grading as described by the International Society of Urological Pathologists (ISUP) and World Health Organization. The highest-grade regions were identified and marked on the slide. Additionally, areas of normal, uninvolved kidney parenchyma were also identified and marked. Corresponding FFPE blocks were then retrieved for both high-grade and non-neoplastic regions. The blocks were compared against the associated marked H\&E stained slides and the corresponding regions were marked on the tissue blocks. $1 \mathrm{~mm}$ punches of tissue were then taken from the blocks.

\section{Whole transcriptome sequencing}

Expression data for 21,111 transcripts were generated using TempO-Seq ${ }^{\mathrm{TM}}$. TempO-Seq was selected, because it does not require RNA extraction from the FFPE, only requires a PCR instrument and a sequencer, and comes with an automated data analysis package for processing the sequencing data and determining differential expression and subtype score, making this assay suitable for use in any research laboratory and in diagnostic and clinical pathology laboratories. TempO-Seq also has the ability to detect low quality and fragmented 
RNA typically found in FFPE samples [14, 15]. Briefly, $1 \mathrm{~mm}$ diameter cores of FFPE renal cancer and adjacent normal tissue were added to a lysis reagent with mineral oil, and then heated at $80^{\circ} \mathrm{C}$ for 5 minutes to deparaffinize the tissue. Tissue was then digested with a protease until fully lysed, then heated to $95^{\circ} \mathrm{C}$ for $15 \mathrm{~min}$ (note that the commercial FFPE kits use a slightly different protocol). A fraction of this lysate was added to a mixture of TempOSeq whole transcriptome Detector Oligos (DOs) which were then hybridized, cleaned up with an exonuclease, and then ligated. The samples were then amplified via PCR using barcoded primers and then pooled into a sequencing library. All reagents were from a prerelease TempO-Seq FFPE assay kit, now commercially available. The sequencing libraries were purified using the Clontech NucleoSpin Gel and PCR Cleanup Kit (Clontech), and sequenced using an Illumina NextSeq, to generate demultiplexed FASTQ files. The libraries were compatible with sequencing on the Illumina platform. Automated TempO-SeqR software was used to align the demultiplexed FASTQ files and produce a read count table for all the samples in the sequencing run, and for other steps, including normalization, averaging of replicates, and calculating averages and statistics for technical replicates without need for expert bioinformatics analysis of the sequencer output.

\section{Statistical analysis}

Independent sample T-test for normally distributed continuous variables, Mann-Whitney U-test for variables that deviate from normality, and chi-squared test were used to understand patients' characteristics. We evaluated if the samples clustered into ccA or ccB using the set of genes identified by Brannon et al [6]. Because of the differences in gene expression platform used by Brannon (microarray) and the targeted sequencing TempO-Seq assay, and differences introduced by using purified RNA for microarrays $v s$ directly assaying lysed FFPE in the TempO-Seq assay, we could not simply compare the expression levels of the signature genes. Instead, a ccA/ ccB score was calculated using a modified algorithm which assigned each sample an A/B score based on whether each gene from the panel was over/under-expressed compared to the average expression for each sample. The final score was the weighted average of the over-expression of group A divided by the weighted average of group B using the coefficients from Brannon et al. Even though of the 103 genes in the Brannon A vs B signature, 85 genes were not differentially expressed in the TempO-Seq data, we kept these in the ratio calculation used to assign subtype. Chisquared tests were used to test the association between molecular subtypes and race/ethnicity as well as between molecular subtypes and pathological characteristics, including SSIGN, and independently, stage, grade, performance status, and necrosis. Association between molecular subtypes and tumor size was tested using the Mann-Whitney U test.

To identify a gene signature associated with outcomes predicted by the SSIGN score, gene expression in aggressive ccRCC (SSIGN score $\geq 7$ ) was compared to that of non-aggressive ccRCC ( $\mathrm{SSIGN} \leq 3$ ). Then, the result was compared to results of differential gene expression analysis for individual clinical characteristics: 1) Fuhrman Grade of 3 or 4 vs. 1 or 2;2) Stage III or IV $v s$. I or II; and 3) necrosis present vs. absent. The DESeq2 differential expression algorithm was used to identify differentially expressed genes [16], and Benjamini and Hochberg's procedure was used to adjust for multiple testing [17]. We considered $P$-values less than 0.05 after adjusting for multiple testing $\left(P_{\mathrm{ADJ}}\right)$ as statistical significant. After $\log _{2}$-transformation, Mann-Whitney U-test and Spearman's correlation was used to evaluate if the top identified genes are correlated with clinical characteristics and outcomes (recurrence and mortality).

TCGA-KIRC level 3 RNA-Seq Upper Quartile normalized FPKM data for ccRCC (451 patients without previous diagnosis of cancer) were used for validation of our findings and to assess associations between expression of identified genes and OS. After $\log _{2}$ transformation, related-samples Wilcoxon Signed Rank Test was used to test if the identified genes were differentially expressed between tumor and normal tissues, and independent samples Mann-Whitney U-test was used to test if identified genes were over-expressed or down-regulated in advanced stage and high-grade tumors. The criteria for validation are differentially expressed between tumor and normal tissues and over-expressed or down-regulated in advanced or high-grade tumors or tumors with necrosis. We considered $P<0.05$ as significant for validation. Survival analysis was also performed using TCGA data. Survival curve was plotted using Kaplan-Meier method, and Log Rank Test as well as Cox regression was used to assess if expression of identified genes predicts OS adjusting for age, gender, race/ethnicity, stage, and grade. Linear trend was examined using the median gene expression of each quartile as a continuous variable.

\section{CONCLUSION}

We demonstrated that an extraction-free transcriptomics assay of FFPE was able to detect existing as well as potentially novel expressed gene biomarkers associated with more aggressive ccRCC. In the current study, only 33 patients were included for discovery of novel gene expression signatures. However, inclusion of a previously underrepresented population in this study identified unique biomarkers that have not been observed or well characterized in previous gene expression studies of ccRCC. Patients with molecular biomarkers of aggressiveness may be counseled towards radical nephrectomy over nephron sparing surgery. 
Author contribution

Research design: Batai K, Ellis EA, Bracamonte E, Lee BR

Data collection: Batai K, Imler E, Bell R, Lwin A, Price E, Milinic T

Data analysis and interpretation: Batai K, Imler E, Pangilinan, Arora A, Seligmann B

Manuscript preparation: All the authors

Manuscript was approved by all the authors

\section{ACKNOWLEDGMENTS}

The authors would like to thank everyone who helped prepare samples and clinical data from Banner University Medical Center, particularly Myra Ortega and University of Arizona Cancer Center Tissue Acquisition and Cellular/Molecular Analysis Shared Resource staff.

\section{CONFLICT OF INTEREST}

This study is a product of a joint effort among the University of Arizona investigators and BioSpyder Technologies, Inc. The University of Arizona investigators contributed to conception of study, collection of data and samples, management of clinical data, and statistical analysis. BioSpyder performed TempO-Seq assay and provided bioinformatic support, including statistical analysis. The University of Arizona investigators and BioSpyder jointly contributed to interpretation of data and manuscript preparation. The University of Arizona investigators did not receive monetary compensation from BioSpyder Technologies, Inc.

\section{FUNDING}

This project was supported by Urology Care Foundation Research Scholar Award, Urology Care Foundation Summer Medical Student Fellowship Program, University of Arizona Department of Surgery Seed Grant, Institutional Research Grant number IRG16-124-37-IRG from the American Cancer Society. This research was also supported through the development of the TempO-Seq FFPE whole transcriptome assay by a grant from the NCI (R33CA183688), grants from NIEHS (R43ES0240107, R44ES024107) grants from NHGRI (R43HG007339, R44HG007339) and an SBIR contract from NCATS (HHSN271201400052C).

\section{REFERENCES}

1. Siegel RL, Miller KD, Jemal A. Cancer statistics, 2017. CA Cancer J Clin. 2017; 67:7-30.

2. Chow W-H, Dong LM, Devesa SS. Epidemiology and risk factors for kidney cancer. Nat Rev Urol. 2010; 7:245-257.
3. Frank I, Blute ML, Cheville JC, Lohse CM, Weaver AL, Zincke H: An outcome prediction model for patients with clear cell renal cell carcinoma treated with radical nephrectomy based on tumor stage, size, grade and necrosis: the SSIGN Score. J Urol. 2002; 168:2395-2400.

4. Parker WP, Cheville JC, Frank I, Zaid HB, Lohse CM, Boorjian SA, Leibovich BC, Thompson RH. Application of the stage, size, grade, and necrosis (SSIGN) score for clear cell renal cell carcinoma in contemporary patients. Eur Urol. 2017; 71:665-673.

5. Chen F, Zhang Y, Şenbabaoğlu Y, Ciriello G, Yang L, Reznik E, Shuch B, Micevic G, De Velasco G, Shinbrot E, Noble MS, Lu Y, Covington KR et al. Multilevel genomicsbased taxonomy of renal cell carcinoma. Cell Rep. 2016; 14:2476-2489.

6. Brannon AR, Reddy A, Seiler M, Arreola A, Moore DT, Pruthi RS, Wallen EM, Nielsen ME, Liu H, Nathanson KL, Ljungberg B, Zhao H, Brooks JD et al. Molecular stratification of clear cell renal cell carcinoma by consensus clustering reveals distinct subtypes and survival patterns. Genes Cancer 2010; 1:152-163.

7. Eckel-Passow JE, Igel DA, Serie DJ, Joseph RW, Ho TH, Cheville JC, Parker AS. Assessing the clinical use of clear cell renal cell carcinoma molecular subtypes identified by RNA expression analysis. Urol Oncol. 2015; 33:68.e17-68. e23.

8. Chen D, Gassenmaier M, Maruschke M, Riesenberg R, Pohla H, Stief CG, Zimmermann W, Buchner A. Expression and prognostic significance of a comprehensive epithelialmesenchymal transition gene set in renal cell carcinoma. J Urol. 2014; 191:479-486.

9. Kosari F, Parker AS, Kube DM, Lohse CM, Leibovich BC, Blute ML, Cheville JC, Vasmatzis G. Clear cell renal cell carcinoma: gene expression analyses identify a potential signature for tumor aggressiveness. Clin Cancer Res. 2005; 11:5128-5139

10. Lane BR, Li J, Zhou M, Babineau D, Faber P, Novick AC, Williams BRG. Differential expression in clear cell renal cell carcinoma identified by gene expression profiling. J Urol. 2009; 181:849-860.

11. Tan W, Hildebrandt MAT, Pu X, Huang M, Lin J, Matin SF, Tamboli P, Wood CG, Wu X. Role of inflammatory related gene expression in clear cell renal cell carcinoma development and clinical outcomes. J Urol. 2011; 186:20712077.

12. Pinheiro PS, Callahan KE, Gomez SL, Marcos-Gragera R, Cobb TR, Roca-Barcelo A, Ramirez AG. High cancer mortality for US-born Latinos: evidence from California and Texas. BMC Cancer. 2017; 17:478.

13. Batai K, Bergersen A, Price E, Hynes K, Ellis NA, Lee BR. Clinical and molecular characteristics and burden of kidney cancer among Hispanics and Native Americans: steps toward precision medicine. Clin Genitourin Cancer. 2018; 16:e535-e541. 
14. Grimm FA, Iwata Y, Sirenko O, Chappell GA, Wright FA, Reif DM, Braisted J, Gerhold DL, Yeakley JM, Shepard P, Seligmann B, Roy T, Boogaard PJ et al. A chemical-biological similarity-based grouping of complex substances as a prototype approach for evaluating chemical alternatives. Green Chem. 2016; 18:4407-4419.

15. Yeakley JM, Shepard PJ, Goyena DE, VanSteenhouse HC, McComb JD, Seligmann BE. A trichostatin A expression signature identified by TempO-Seq targeted whole transcriptome profiling. PLOS ONE. 2017; 12:e0178302.

16. Love MI, Huber W, Anders S. Moderated estimation of fold change and dispersion for RNA-seq data with DESeq2. Genome Biol. 2014; 15:550.

17. Benjamini Y, Hochberg Y. Controlling the false discovery rate: a practical and powerful approach to multiple testing. J R Stat Soc Ser B Methodol. 1995; 57:289-300.

18. Yao M, Huang Y, Shioi K, Hattori K, Murakami T, Sano F, Baba M, Kondo K, Nakaigawa N, Kishida T, Nagashima J, Yamada-Okabe H, Kubota Y. A three-gene expression signature model to predict clinical outcome of clear cell renal carcinoma. Int J Cancer. 2008; 123:1126-1132.

19. Wozniak MB, Le Calvez-Kelm F, Abedi-Ardekani B, Byrnes G, Durand G, Carreira C, Michelon J, Janout V, Holcatova I, Foretova L, Brisuda A, Lesueur F, Mckay J et al. Integrative genome-wide gene expression profiling of clear cell renal cell carcinoma in Czech Republic and in the United States. PLOS ONE. 2013; 8:e57886.

20. Thibodeau BJ, Fulton M, Fortier LE, Geddes TJ, Pruetz BL, Ahmed S, Banes-Berceli A, Zhang PL, Wilson GD, Hafron J. Characterization of clear cell renal cell carcinoma by gene expression profiling. Urol Oncol. 2016; 34:168.e161168.e169.

21. Cheng J, Zhang J, Han Y, Wang X, Ye X, Meng Y, Parwani A, Han Z, Feng Q, Huang K. Integrative analysis of histopathological images and genomic data predicts clear cell renal cell carcinoma prognosis. Cancer Res. 2017; 77:e91-e100.

22. Pandey P, Sliker B, Peters HL, Tuli A, Herskovitz J, Smits K, Purohit A, Singh RK, Dong J, Batra SK, Coulter DW, Solheim JC. Amyloid precursor protein and amyloid precursor-like protein 2 in cancer. Oncotarget. 2016; 7:19430-19444.

23. Brindley, DN. Lipid phosphate phosphatases and related proteins: signaling functions in development, cell division, and cancer. J Cell Biochem. 2004; 92:900-912.

24. Zhang Q, Yang Z, Han Q, Bai H, Wang Y, Yi X, Yi Z, Yang L, Jiang L, Song X, Kuang Y, Zhu Y. G6PD promotes renal cell carcinoma proliferation through positive feedback regulation of p-STAT3. Oncotarget. 2017; 8:109043109060.

25. Zhang Q, Yi X, Yang Z, Han Q, Di X, Chen F, Wang Y, Yi Z, Kuang Y, Zhu Y. Overexpression of G6PD represents a potential prognostic factor in clear cell renal cell carcinoma. J Cancer. 2017; 8:665-673.

26. Ju HQ, Lu YX, Wu QN, Liu J, Zeng ZL, Mo HY, Chen Y, Tian T, Wang Y, Kang TB, Xie D, Zeng MS, Huang P et al. Disrupting G6PD-mediated Redox homeostasis enhances chemosensitivity in colorectal cancer. Oncogene. 2017; 36:6282-6292.

27. González-Vallinas M, Vargas T, Moreno-Rubio J, Molina S, Herranz J, Cejas P, Burgos E, Aguayo C, Custodio A, Reglero G, Feliu J, Ramírez de Molina A. Clinical relevance of the differential expression of the glycosyltransferase gene GCNT3 in colon cancer. Eur J Cancer. 2015; 51:1-8.

28. Rao CV, Janakiram NB, Madka V, Kumar G, Scott EJ, Pathuri G, Bryant T, Kutche H, Zhang Y, Biddick L, Gali H, Zhao Y, Lightfoot S et al: Small-molecule inhibition of GCNT3 disrupts mucin biosynthesis and malignant cellular behaviors in pancreatic cancer. Cancer Res. 2016; 76:19651974.

29. Calle EE, Rodriguez C, Walker-Thurmond K, Thun MJ. Overweight, obesity, and mortality from cancer in a prospectively studied cohort of U.S. adults. N Engl J Med. 2003; 348:1625-1638.

30. Heymsfield SB, Peterson CM, Thomas DM, Heo M, Schuna JM. Why are there race/ethnic differences in adult body mass index-adiposity relationships? A quantitative critical review. Obes Rev. 2016; 17:262-275.

31. Gati A, Kouidhi S, Marrakchi R, El Gaaied A, Kourda N, Derouiche A, Chebil M, Caignard A, Perier A. Obesity and renal cancer. OncoImmunology. 2014; 3:e27810.

32. Chevrier S, Levine JH, Zanotelli VRT, Silina K, Schulz D, Bacac M, Ries CH, Ailles L, Jewett MAS, Moch H, van den Broek M, Beisel C, Stadler MB et al. An immune atlas of clear cell renal cell carcinoma. Cell. 2017; 169:736-749. e718. 